\title{
McConnell's sign; a distinctive echocardiographic finding for diagnosing acute pulmonary embolism in emergency department
}

\author{
Seong Beom Oh*, Seung Jae Bang, Min Jeong Kim \\ From 10th WINFOCUS World Congress on Ultrasound in Emergency and Critical Care \\ Kuala Lumpur, Malaysia. 16-19 November 2014
}

\section{Background}

McConnell's sign, that is regional RV dysfunction, with akinesia of the mid free wall but normal motion at the apex is a distinct echocardiographic finding described in patients with acute pulmonary embolism.

\section{Objective}

The aim of this prospective study was to analyze clinical usefulness of McConnell's sign for diagnosing acute pulmonary embolism before computed tomography scan in emergency department.

\section{Patients and methods}

From June 2013 through August 2014, 15 patients who were observed McConnell's sign from bedside echocardiography performed by emergency medicine specialist were enrolled in this study. Those whose final diagnosis was acute pulmonary embolism were classified into three groups such as main, lobar and segmental pulmonary artery thrombus group according to the thrombus location based on the CT scan. Demographic and various clinical data were recorded and analyzed.

\section{Results}

Average age was $64.7 \pm 15.6$ and female was $9(60 \%)$. In 15 , final diagnosis of two subjects was not acute pulmonary embolism. One was acute respiratory distress syndrome and the other was inferior wall STEMI with RV infarction. The rest of the subjects( 13 of $15,86.7 \%$ ) were diagnosed acute pulmonary embolism with CT scan. In these subjects, 12(92.3\%) were identified as huge thrombus in main pulmonary artery and the other in lobar pulmonary artery.
There was no subject whose thrombus was identified in segmental pulmonary artery.

\section{Conclusion}

Regional RV dysfunction known as McConnell's sign was very distinctive echocardiographic finding for diagnosing acute pulmonary embolism in emergency department. In addition, identification of this sign strongly suggests massive thrombus burden such as main pulmonary artery thrombus rather than lobar or segmental artery.

Published: 9 March 2015

\section{References}

1. McConnell MV, Solomon SD, Rayan ME, Come PC, Goldhaber SZ, Lee RT: Regional right ventricular dysfunction detected by echocardiography in acute pulmonary embolism. Am J Cardiol 1996, 78:469-73.

2. Casazza F, Bongarzoni A, Capozi A, Agostoni O: Regional right ventricular dysfunction in acutepulmonary embolism and right ventricular infarction. Eur J Echocardiogr 2005, 6:11-4.

3. Rudski $L G$, et al: Guideline for the echocardiographic assessment of the right heart in adults: a report from the American society of echocardiography. J Am soc Echocardiogr 2010, 23:685-713.

doi:10.1186/2036-7902-7-S1-A20

Cite this article as: Oh et al:: McConnell's sign; a distinctive echocardiographic finding for diagnosing acute pulmonary embolism in emergency department. Critical Ultrasound Journal 2015 7(Suppl 1):A20.

* Correspondence: holytiger@hanmail.net

Department of Emergency Medicine, College of Medicine, Dankook

University, Cheonan, Republic of Korea

(c) 2015 Oh et al; licensee Springer. This is an Open Access article distributed under the terms of the Creative Commons Attribution License (http://creativecommons.org/licenses/by/4.0), which permits unrestricted use, distribution, and reproduction in any medium, provided the original work is properly cited. 